\title{
Mesoporous silica supported Pd/Ag bimetallic nanoparticles as a plasmonic catalyst for chemoselective hydrogenation of $p$-nitrostyrene under visible light irradiation
}

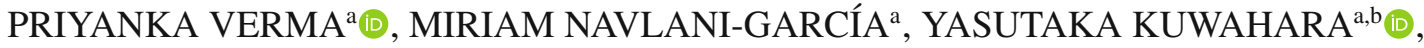 \\ KOHSUKE MORI $^{\mathrm{a}, \mathrm{b}, \mathrm{c}} \mathbb{D}$ and HIROMI YAMASHITA ${ }^{\mathrm{a}, \mathrm{b}, *} \mathbb{D}$ \\ a Division of Materials and Manufacturing Science, Graduate School of Engineering, Osaka University, \\ 2-1 Yamada-oka, Suita, Japan \\ ${ }^{\mathrm{b}}$ Unit of Elements Strategy Initiative for Catalysts and Batteries, Kyoto University, Katsura, Kyoto 615-8520, \\ Japan \\ c JST, PRESTO, 4-1-8 Honcho, Kawaguchi, Saitama, Japan \\ E-mail: yamashita@mat.eng.osaka-u.ac.jp
}

MS received 12 April 2017; revised 14 July 2017; accepted 17 July 2017; published online 19 September 2017

\begin{abstract}
Silver-based bimetallic Pd/Ag plasmonic catalysts supported on mesoporous silica catalyze the chemoselective reduction of nitrostyrene (NS) to aminostyrene (AS). Ammonia borane (AB) is used as a mild reducing agent to generate $\mathrm{H}_{2}$ in situ on the surface of exposed $\mathrm{Ag}$ and $\mathrm{Pd}$ atoms for $-\mathrm{NO}_{2}$ reduction at room temperature and atmospheric pressure. Systematic studies on the effect of nanoparticles (NPs) size are performed in dark and under light irradiation conditions. The spherical yellow colored bimetallic Pd/Ag/SBA-15 (Y) exhibited maximum AS conversion of $89 \%$ at $90 \mathrm{~min}$ under visible light irradiation. The catalysts display higher selectivity under visible light irradiation which can be due to the efficient adsorption and transfer of $\mathrm{H}^{+} / \mathrm{H}^{-}$pair to the polar bonds in the nitro group.
\end{abstract}

Keywords. Ag-plasmon; visible light irradiation; chemoselective hydrogenation; bimetallic; mesoporous silica.

\section{Introduction}

In the recent years, catalysis has received considerable attention utilizing light as an energy source for carrying out solar-to-chemical conversion catalytic reactions. The substantial significance and applications of plasmonic photocatalysts have led to the tremendous efforts and rapid developments in this branch of photocatalysis during the last few years. Metal nanostructures based heterogeneous photocatalyst is being extensively studied to design visible light sensitive composite materials owing to their localized surface plasmonic resonance effect. ${ }^{1}$ Localized surface plasmon resonance (LSPR) can be defined as the coherent oscillation of the metalfree electrons in the presence of an external electric field. The size and shape of the plasmonic nanoparticles (NPs) are considered to be of significant importance in the excitation and hot electron generation. ${ }^{2}$ The resonance absorption wavelength, intensity and the efficiency of charge separation process are distinctly affected by tailoring the size, morphology and surrounding medium of the metal nanostructures. For example, the absorption wavelength of the sphere, rod and cube represent one, two and three peaks in the absorption spectrum, respectively. ${ }^{3}$ Different morphologies of NPs give rise to different polarization modes and hence different number of peaks in the spectrum, by interacting with incident light irradiation. ${ }^{4}$ The spectrum exhibits red-shift on increasing the size of nanoparticles. The morphology controlled Ag NPs have been exploited for its use in the selective hydrogenation of nitrostyrene under visible light irradiation.

Chemoselective hydrogenation of nitroaromatics having other reducible functionalities (like $\mathrm{C}=\mathrm{O}$ and $\mathrm{C}=\mathrm{C}$ )

\footnotetext{
*For correspondence

Electronic supplementary material: The online version of this article (https://doi.org/10.1007/s12039-017-1364-5) contains supplementary material, which is available to authorized users.
} 
is an important and challenging chemical reaction for the formation of various important intermediates in industries like agrochemicals, pharmaceuticals, polymers, herbicides and dyes. ${ }^{5-7}$ A stoichiometric amount of $\mathrm{Sn}, \mathrm{Zn}$ or Fe can also carry out the same process but by generating large amounts of harmful wastes, acid additives and low atom efficiencies. Although being important, only a few reports have been published, till date, in the selective hydrogenation of nitrostyrene in the presence of $\mathrm{C}=\mathrm{C}$ or $\mathrm{C}=\mathrm{O}$ bonds.$^{5,8-10}$ The initial successful studies were performed by Corma et al., utilizing $\mathrm{Au}$ NPs on $\mathrm{TiO}_{2}$ and $\mathrm{Fe}_{2} \mathrm{O}_{3}$ with $\mathrm{H}_{2}$ gas under mild reaction conditions. ${ }^{11,12}$ It would be more interesting to execute the reaction using reducing agents other than gaseous $\mathrm{H}_{2}$ and $\mathrm{CO}$ utilizing metal NPs as a catalyst at room temperature and atmospheric pressure conditions. ${ }^{13-15}$ Our group has reported the tandem hydrogenation reaction of $p$-nitrophenol to $p$-aminophenol using Ag-based catalyst and $\mathrm{AB}$ as the source of in situ $\mathrm{H}_{2} .{ }^{16}$ From theoretical and experimental revelations, $\mathrm{Ag}$ NPs is known to have very weak interaction with $\mathrm{H}_{2}$ because of its fullyfilled d-orbitals, but recently, it has been realized that $\mathrm{Ag}$ NPs, can also serve as heterogeneous catalysts in liquid phase reactions. Heterolytic cleavage of $\mathrm{H}_{2}$ is favored for the reduction of the polar nitro group in preference to the nonpolar $\mathrm{C}=\mathrm{C}$ bonds. The formation of $\mathrm{H}^{+} / \mathrm{H}^{-}$ pair transfers preferentially to the polar bonds leading to chemoselective reduction. ${ }^{5,17,18}$

Mesoporous silica supported metal nanoparticles are desired for various applications because of their stability, efficient adsorption, metal-support interaction and tuning the size and morphology of metal nanoparticles. ${ }^{19}$ Size and color controlled $\mathrm{Ag}$ and $\mathrm{Pd} / \mathrm{Ag}$ coreshell structure are supported on mesoporous silica for tuning their size, morphology and enhanced absorption and stability. ${ }^{20}$ We have utilized the plasmonic effect of Ag nanostructures which are capable of converting solar energy into chemical energy by localized surface plasmon resonance (LSPR). ${ }^{21-23}$ Significant enhancements in the selectivity and conversions under visible light irradiation were observed. $\mathrm{Pd} / \mathrm{Ag}$ catalysts showed superior performance than monometallic Ag catalysts.

Our group has published some reports on the synthesis and catalytic plasmonic performance of $\mathrm{Ag}, \mathrm{Pd} / \mathrm{Ag}$, $\mathrm{Pd} / \mathrm{Au}, \mathrm{Ag} / \mathrm{Ti}, \mathrm{Pt} / \mathrm{Ag}$ and $\mathrm{M} / \mathrm{Ag}$ nanorods $(\mathrm{M}=\mathrm{Ru}$, $\mathrm{Ni}, \mathrm{Co}, \mathrm{Pd})$ in various chemical reactions. ${ }^{24-27}$ In the present study, the size and color controlled $\mathrm{Ag}$ and $\mathrm{Pd} / \mathrm{Ag}$ NPs supported on mesoporous silica are explored for the chemoselective reduction of $p$-nitrostyrene ( $p$ $\mathrm{NS}$ ) to $p$-aminostyrene ( $p$-AS) using $\mathrm{AB}$ as a mild reducing agent and to observe the enhancements and improved selectivity under visible light irradiation conditions.

\section{Experimental}

\subsection{Materials}

Tetraethyl orthosilicate $\left.\left(\left(\mathrm{C}_{2} \mathrm{H}_{5} \mathrm{O}\right)_{4} \mathrm{Si}\right)\right)$, hydrochloric acid ( $\mathrm{HCl})$, 1-hexanol $\left(\mathrm{C}_{6} \mathrm{H}_{13} \mathrm{OH}\right)$, acetone, silver nitrate $\left(\mathrm{AgNO}_{3}\right)$, sodium laurate, and ethanol were purchased from Nacalai Tesque Inc. Triblock Pluronic $\mathrm{P} 123^{\circledR}\left(\mathrm{M}_{w}=5800, \mathrm{PEO}_{20}\right.$ $\left.\mathrm{PPO}_{70} \mathrm{PEO}_{20}\right)$ and ammonia borane $\left(\mathrm{NH}_{3} \mathrm{BH}_{3}\right)$ were obtained from Sigma-Aldrich Co. 4-nitrostyrene $\left(\mathrm{C}_{8} \mathrm{H}_{7} \mathrm{NO}_{2}\right)$ was purchased from Tokyo Chemical Industry Co. Ltd. All chemicals were used as received without any further purification.

\subsection{Preparation of SBA-15}

Mesoporous silica SBA-15 was prepared according to the method reported in the literature, using Pluronic P123 ${ }^{\circledR}$ as a structure directing agent and tetraethyl orthosilicate as a silica source under acidic conditions $(\mathrm{pH}<1)$. Briefly, the P123 polymer was dissolved in water and $0.1 \mathrm{~N} \mathrm{HCl}$ with continuous stirring at $40^{\circ} \mathrm{C}$ for $3 \mathrm{~h}$ at $400 \mathrm{rpm}$. The source of silica, TEOS, was slowly added to the reaction mixture under vigorous stirring at $40^{\circ} \mathrm{C}$ for $24 \mathrm{~h}$. The suspension was transferred to the Teflon bottle after $24 \mathrm{~h}$ and aged at $80^{\circ} \mathrm{C}$ for $24 \mathrm{~h}$ in air. The obtained slurry was then filtered, washed with copious amount of deionized water and dried at $80^{\circ} \mathrm{C}$ overnight. The obtained white solid was finally calcined in air at $550^{\circ} \mathrm{C}$ for $5 \mathrm{~h}$ in order to remove the organic template. The white colored powder was used as a support to synthesize metal nanoparticles onto it.

\subsection{Preparation of $A g / S B A-15$}

$1 \mathrm{wt} . \%$ of Ag nanoparticles was incorporated within the mesoporous channels of SBA-15. In a typical procedure, SBA-15 $(0.396 \mathrm{~g})$ is dispersed in 1-hexanol $(40 \mathrm{~mL})$ and ultrasonicated till it becomes a clear solution. An aqueous solution of $\mathrm{AgNO}_{3}(0.037 \mathrm{mmol})$ and sodium laurate $(10 \mathrm{mg})$ as a surface modifying ligand was added to the above solution under an $\mathrm{Ar}$ atmosphere. The solution was then irradiated by microwave $(500 \mathrm{~W}, 2450 \pm 30 \mathrm{MHz})$ for different periods of time ( 3 or $5 \mathrm{~min}$ ), with or without a surface modifying agent. The resultant solution was filtered and washed with distilled water and acetone, followed by drying at $80^{\circ} \mathrm{C}$ in air overnight. Three different-colored Ag/SBA-15 with different morphologies were synthesized by varying the microwave irradiation time. The obtained yellow, red, and blue Ag catalysts were denoted as Ag/SBA-15 (Y), Ag/SBA-15 (R), and Ag/SBA-15 (B), respectively.

\subsection{Preparation of $\mathrm{Pd} / \mathrm{Ag} / \mathrm{SBA}-15$}

0.1 wt $\%$ of Pd was deposited onto Ag/SBA- 15 by plasmonmediated deposition method under visible light irradiation. $0.1 \mathrm{~g}$ of $\mathrm{Ag} / \mathrm{SBA}-15$ was suspended and ultrasonicated in water $(20 \mathrm{~mL})$ until it became a clear solution. It was followed by bubbling Ar gas for a period of $30 \mathrm{~min}$ to ensure complete 

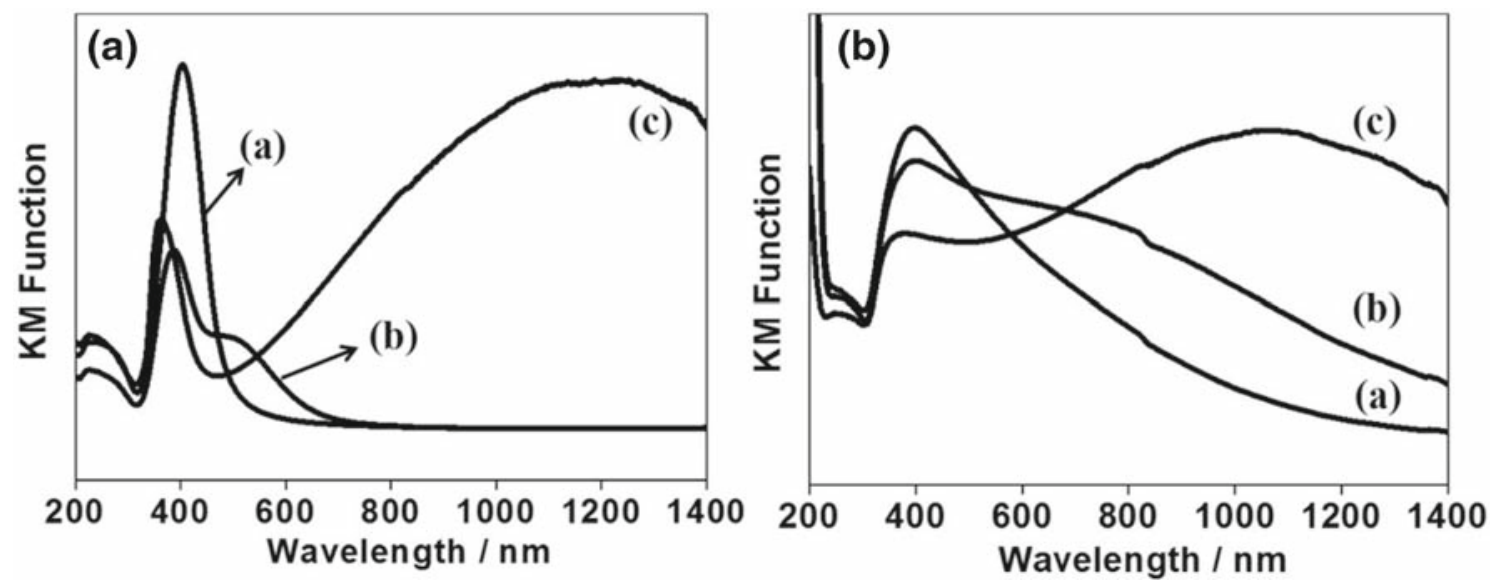

Figure 1. UV-vis spectra of (A) (a) Ag/SBA-15 (Y), (b) Ag/SBA-15 (R), (c) Ag/SBA-15 (B) and (B) (a) Pd/Ag/SBA-15 (Y), (b) Pd/Ag/SBA-15 (R), (c) Pd/Ag/SBA-15 (B).

inert atmosphere. Subsequently, $0.482 \mathrm{~mL}$ of $\mathrm{Pd}(\mathrm{OAc})_{2}$ solution $(1.93 \mathrm{mM})$ was injected into the reaction mixture and irradiated with a Xe lamp (500 W; San-Ei Electric Co. Ltd. XEF-501S) with continuous stirring for $2 \mathrm{~h}$. The resultant mixture was filtered, washed with distilled water and dried at $80^{\circ} \mathrm{C}$ in air overnight. The obtained catalysts were denoted as $\mathrm{Pd} / \mathrm{Ag} / \mathrm{SBA}-15(\mathrm{Y}), \mathrm{Pd} / \mathrm{Ag} / \mathrm{SBA}-15(\mathrm{R})$, and Pd/Ag/SBA-15 (B), respectively.

\subsection{Catalytic reaction}

Chemoselective reduction of $p$-nitrostyrene ( $p$-NS) to $p$ aminostyrene ( $p$-AS): At first, the $5 \mathrm{mg}$ of catalyst was measured and dispersed in Pyrex vessel sealed with rubber septum followed by Ar bubbling for 30 min to maintain an inert atmosphere. The separate solutions of $20 \mathrm{mM} p$-NS and $0.06 \mathrm{M}$ ammonia borane $(\mathrm{AB})$ in ethanol was also prepared. $5 \mathrm{~mL}$ of each solution ( $p$-NS and $\mathrm{AB}$ ) was injected through the rubber septum and stirred continuously in dark or under light irradiation conditions. An external fan was used in the reaction system to maintain the constant temperature of reaction vessel during the course of the reaction. The products formed were detected and monitored by using Shimadzu GC-2010 chromatograph installed with Shimadzu Mass spectrometer GC-MS-QP2010 Plus at fixed intervals of time.

\subsection{Characterization}

The structural characteristics of $\mathrm{Ag}$ and $\mathrm{Pd} / \mathrm{Ag}$ supported on SBA-15 silica were substantiated by UV-vis, $\mathrm{N}_{2}$ physisorption, transmission electron microscopy (TEM), X-ray photoelectron spectroscopy (XPS) and extended X-ray absorption fine structure (EXAFS). The reflectance UV-Vis spectra of powdered samples were obtained by using Shimadzu UV2450 spectrophotometer. $\mathrm{BaSO}_{4}$ was used as a standard material and the absorption spectra were collected by using the Kubelka-Munk function. Nitrogen adsorption studies were performed by using BEL-SORP max system (MicrotraBEL
Corp.) at $-196^{\circ} \mathrm{C}$. In order to remove the adsorbed impurities, the samples were degassed in vacuum at $200^{\circ} \mathrm{C}$ for $3 \mathrm{~h}$ prior to analysis. TEM micrographs were obtained by using Hitachi Hf-2000 field emission-transmission electron microscope (FE-TEM) equipped with Kevex energy-dispersive $\mathrm{X}$-ray detector operated at $200 \mathrm{kV}$. Ag K-edge and Pd Kedge X-ray absorption fine structure (XAFS) spectra were recorded by fluorescence-yield collection technique at beam line 01B1 station with an attached Si (111) monochromator at SPring-8, JASRI, Harima, Japan (Prop. No. 2015A1149). ESCA-3400 electron spectrometer was used to characterize samples for X-ray photoelectron spectroscopy.

\section{Results and Discussion}

\subsection{Characterization results}

3.1a UV-vis absorption measurement: In this study, the size and color controlled Ag NPs responded differently with the visible light irradiation as shown in Figure 1 (A). Ag NPs with spherical (yellow), shorter aspect ratio rods (red) and longer aspect ratio rods (blue) showed characteristics plasmonic peak $(\lambda=420 \mathrm{~nm})$ in the UV-vis spectrum. With an objective to improve the activity of $\mathrm{Ag}$ plasmonic photocatalyst, minimal amounts of an active transition metal, Pd was deposited onto it. The absorption behavior of the resultant catalysts is shown in Figure 1 (B). The absorption intensity found out to be decreased and spectral peak became a little broader for the three catalysts, which might be due to a surface coverage of plasmonic Ag NPs.

3.1b TEM measurement: The TEM micrographs of three different Ag NPs are shown in Figure 2. It can be observed that morphology controlled Ag NPs are grown within the channels of mesoporous silica as observed 

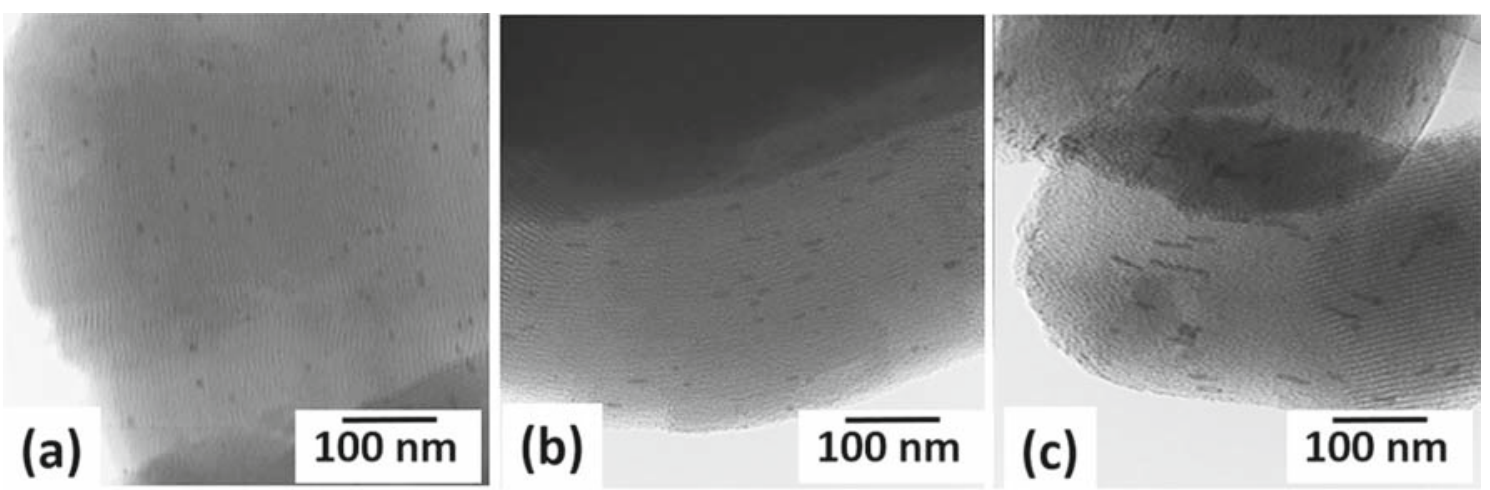

Figure 2. TEM images (a) Ag/SBA-15 (Y), (b) Ag/SBA-15 (R) and (c) Ag/SBA-15 (B).

by the $\mathrm{BJH}$ mesopore volume analysis. $\mathrm{N}_{2}$ physisorption analysis showed the decreased mesoporous volume of silica upon metal NPs incorporation confirming the above observation. Ag/SBA-15 (Y) found to be spherical, Ag/SBA-15 (R) with rod-like morphology and Ag/SBA-15 (B) was rod-like with longer aspect ratio. The average size of yellow Ag/SBA-15 (Y) NPs was found to be $5 \mathrm{~nm}$, red colored Ag/SBA-15 (R) length of $\sim 20 \mathrm{~nm}$, and blue Ag/SBA-15 (B) of an approximate length of $34 \mathrm{~nm}$. The sample images of Ag NPs and $\mathrm{Pd} / \mathrm{Ag}$ NPs is shown in Figure S1 (a-f) (Supplementary Information). A TEM and HAADF-STEM of Ag/SBA-15 (B) is also shown in Figure S2 (Supplementary Information) to confirm the growth of metal nanoparticles within the mesoporous channels of silica. After depositing Pd NPs onto Ag, there was no substantial change in the morphology of NPs. The TEM micrographs along with sample images (inset) for $\mathrm{Pd}$ deposited Ag NPs is shown in Figure S3 (Supplementary Information). The color of the samples changed drastically after depositing Pd from yellow, red and blue to greyish-black for all the catalysts. The bimetallic heterojunction is difficult to observe because of very low metal loadings of Pd (0.1 wt.\%) on Ag. The catalytic activities were found to be significantly dependent on the morphology and showed significant enhancement upon Pd deposition as discussed in section 3.2.

3.1c XAFS analysis: The electronic structure and chemical environment of $\mathrm{Ag}$ and $\mathrm{Pd} / \mathrm{Ag}$ bimetallic NPs, were studied by extended X-ray absorption measurements. Figure S4 (Supplementary Information) shows the Fourier transformation (FT) of $k^{3}$-weighted extended X-ray absorption fine structure (EXAFS) of Ag K- and Pd K-edge. Figure S4 (Supplementary Information) (A) summarizes the Ag K-edge FT-XAFS spectra for AgO, Ag foil, Ag/SBA-15 (B) and Pd/Ag/SBA15 (B). A single peak at $2.7 \AA$ for $\mathrm{Ag}$ foil representing $\mathrm{Ag}-\mathrm{Ag}$ contiguous bond in metallic form. The peak shown by $\mathrm{Ag} / \mathrm{SBA}-15$ and $\mathrm{Pd} / \mathrm{Ag} / \mathrm{SBA}-15$ similar to $\mathrm{Ag}$ foil but different from $\mathrm{AgO}$ confirms the metallic nature in the samples. The Pd K-edge XAFS spectra is shown in Figure $\mathrm{S} 4$ (B) for Pd/Ag/SBA-15 (B) catalyst, $\mathrm{Pd}$ foil and $\mathrm{PdO}$ as standard materials. The bimetallic Pd/Ag/SBA-15 spectra similar to Pd foil confirms the Pd-Pd metallic bonding. The obvious different peaks shown by $\mathrm{PdO}$ shows the absence of oxide species in the prepared catalysts.

3.1d XPS analysis: X-ray photoelectron spectroscopy was utilized to characterize the surface sensitivity and elemental composition of prepared samples. Figure 3A displays the $\mathrm{Ag} 3 \mathrm{~d}$ core electrons for $\mathrm{Ag} / \mathrm{SBA}-15$ and Pd/Ag/SBA-15 (Y), (R), (B) NPs supported on mesoporous silica. The observed standard peaks from the spectra for $\mathrm{Ag}^{0}$ observed around $367.3 \mathrm{eV}$ and $373.3 \mathrm{eV}$ for $3 d_{5 / 2}$ and $3 d_{3 / 2}$, respectively. Figure $3 B$ represents the $\mathrm{Pd} 3 \mathrm{~d}$ core electrons of $\mathrm{Pd} / \mathrm{Ag} / \mathrm{SBA}-15(\mathrm{Y}),(\mathrm{R})$ and $(\mathrm{B})$. The metallic Pd shows standard peaks at $334.4 \mathrm{eV}$ and $340.4 \mathrm{eV}$ for $3 \mathrm{~d}_{5 / 2}$ and $3 \mathrm{~d}_{3 / 2}$, respectively. The presence of doublet peaks $3 \mathrm{~d}_{3 / 2}$ and $3 \mathrm{~d}_{5 / 2}$ separated by $6.0 \mathrm{eV}$ for $\mathrm{Ag}$ and $\mathrm{Pd}$ validates the surface composition and zero valent state of prepared samples. The smoothing of less intense peaks for Pd $3 \mathrm{~d}$ spectra was done due to the minimal loading amounts of $\mathrm{Pd}(0.1 \mathrm{wt} . \%)$ on $\mathrm{Ag}$ in the catalytic system.

\subsection{Catalytic reaction}

The chemoselective reduction of nitro to amino group in the presence of reducible groups like $\mathrm{C}=\mathrm{C}$ bond is still a challenge to be achieved. ${ }^{28-31}$ The catalytic activity of $\mathrm{Ag}$ and $\mathrm{Pd} / \mathrm{Ag}$ NPs was evaluated in the chemoselective hydrogenation of $p$-nitrostyrene to $p$-aminostyrene using $\mathrm{AB}$ (hydrogen source) as a reducing agent as shown in Scheme 1. AB is a white colored solid at room temperature which contains $19.6 \mathrm{wt}$ \% of hydrogen and has attracted great attention to be used as a 

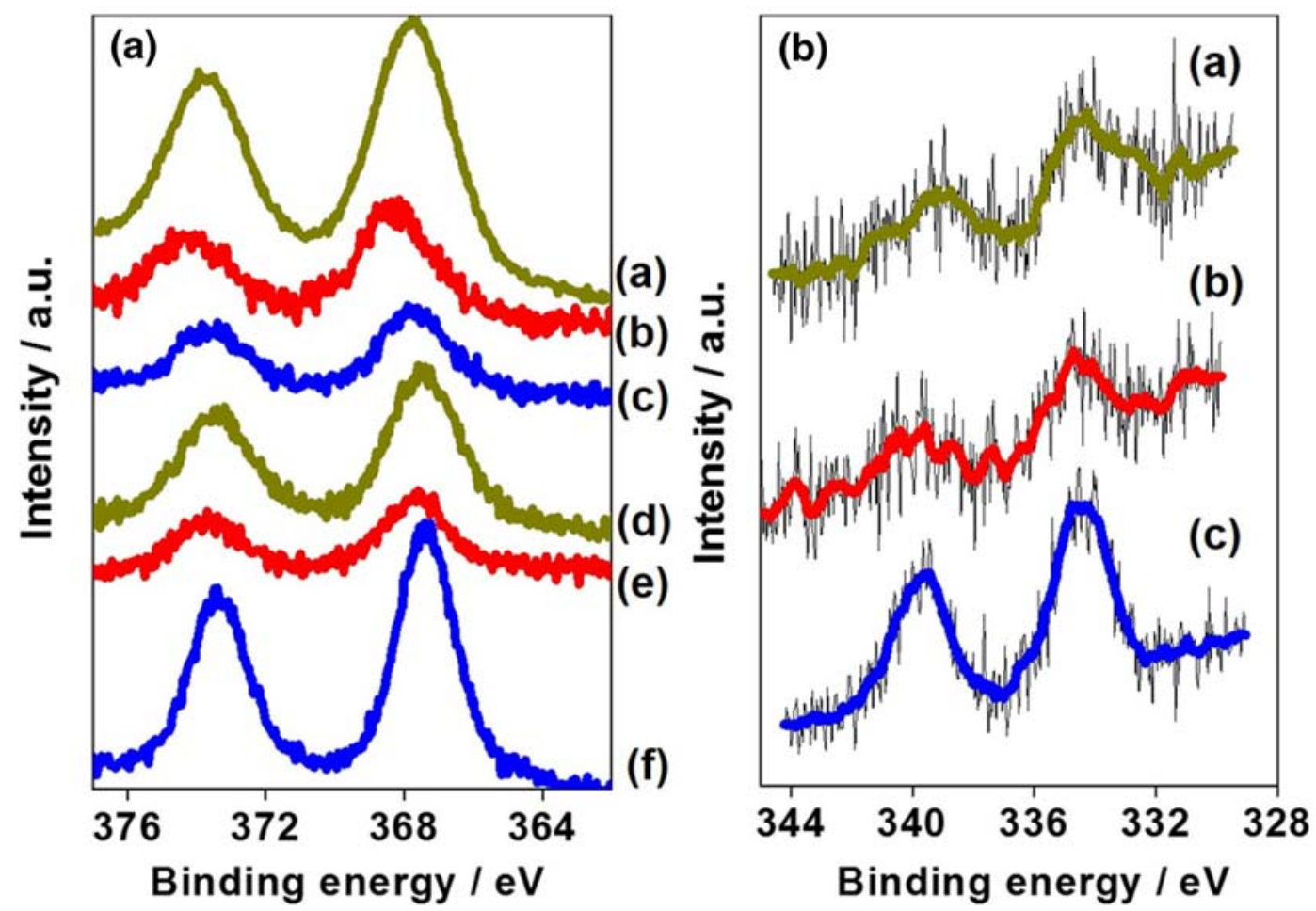

Figure 3. XPS spectra of (A) Ag 3d for (a) Ag/SBA-15 (Y), (b) Ag/SBA-15 (R), (c) Ag/SBA-15 (B), (d) Pd/Ag/SBA-15 (Y), (e) Pd/Ag/SBA-15 (R), (f) Pd/Ag/SBA-15 (B) and (B) Pd 3d spectra for (a) Pd/Ag/SBA-15 (Y), (b) Pd/Ag/SBA-15 (R), (c) d/Ag/SBA-15 (B).

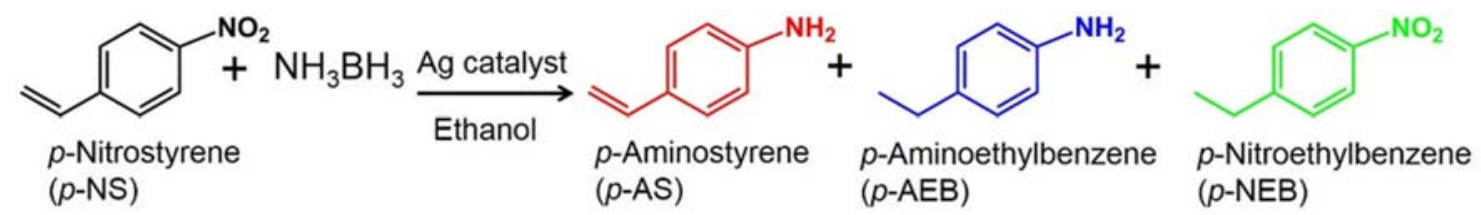

Scheme 1. Reduction of $p$-nitrostyrene over Ag catalysts using ammonia borane as a source of $\mathrm{H}_{2}$ in ethanol aqueous solution.

promising storage material in the recent past. ${ }^{32,39,40}$ The three products observed in major proportions during the reduction reaction of $p$-nitrostyrene ( $p$-NS) are $p$ aminostyrene ( $p$-AS), $p$-aminoethyl benzene ( $p$-AEB) and $p$-nitroethylbenzene ( $p$-NEB). $p$-AS is the main and desired product, while $p$-AEB and $p$-NEB form by the complete and incomplete reduction of $p$-NS, respectively. The reaction was carried out in dark and under light irradiation conditions to study the effect of plasmonic NPs in the selective catalysis.

The reduction of nitro to amino group takes place via formation of nitroso and hydroxylamines intermediates as shown in the following equation (1). ${ }^{33-38}$ However, the intermediates are difficult to be detected due to their fast conversion rates.

$$
\begin{aligned}
& \mathrm{R}-\mathrm{NO}_{2}+2 \mathrm{e}^{-} \longrightarrow \mathrm{R}-\mathrm{NO} \\
& +2 \mathrm{e}^{-} \longrightarrow \mathrm{R}-\mathrm{NH}(\mathrm{OH}) \\
& +2 \mathrm{e}^{-} \longrightarrow \mathrm{R}^{-\mathrm{NH}_{2}}
\end{aligned}
$$

The catalytic reaction time profiles of all catalysts in dark conditions are shown in Figure 4. None of the products were observed in the absence of catalysts. The monometallic Ag NPs exhibited less activity and selectivity than bimetallic NPs. The catalytic activity follows the following order; $\mathrm{Pd} / \mathrm{Ag} / \mathrm{SBA}-15(\mathrm{Y})>\mathrm{Pd} / \mathrm{Ag} / \mathrm{SBA}$ $15(\mathrm{R})>\mathrm{Pd} / \mathrm{Ag} / \mathrm{SBA}-15$ (B) $>$ Ag/SBA-15 (Y) > Ag/SBA-15 (R) > Ag/SBA-15 (B). Pd deposition led to the significant improvement in the catalytic activity and $p$-AS selectivity among other products. $\mathrm{Pd} / \mathrm{Ag} / \mathrm{SBA}$ 15 (Y) displayed the highest conversion amount of $68 \%$ $p-\mathrm{AS}$ at $90 \mathrm{~min}$, while the least amount of $9.3 \%$ conversion was observed for Ag/SBA-15 (B). The smallest size Ag/SBA-15 (Y) and Pd/Ag/SBA-15 (Y) nanoparticles were more efficient than larger size nanorods, giving us the evidence that reaction takes place on the exposed surface atoms of Ag NPs. This size dependence on the catalytic reaction activity was also observed in the rate of hydrogen production from $\mathrm{AB}$, as reported earlier. ${ }^{24}$ 

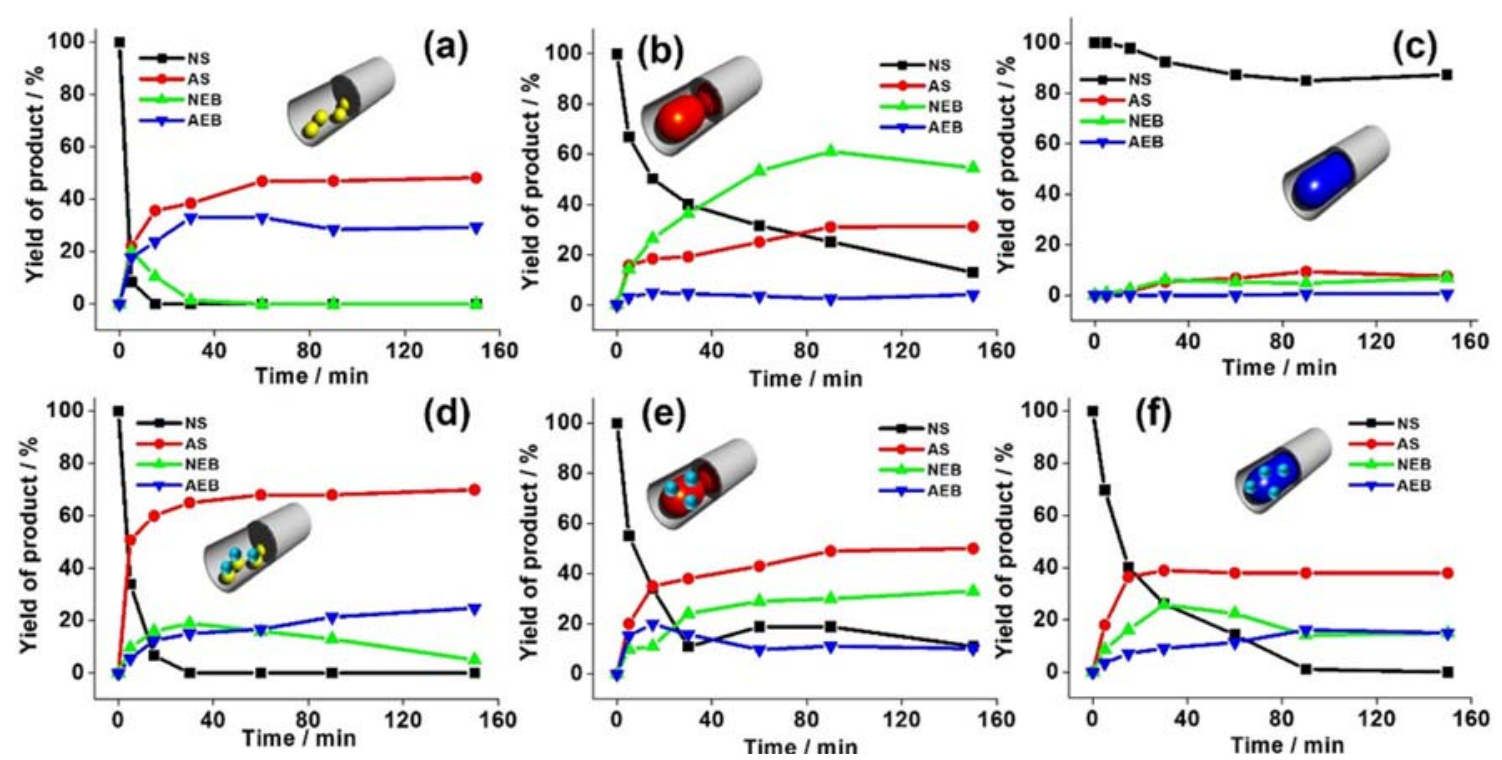

Figure 4. Chemoselective hydrogenation reaction of $p$-nitrostyrene ( $p$-NS) to $p$-aminostyrene ( $p$-AS) using (a) $\mathrm{Ag} / \mathrm{SBA}-15$ (Y), (b) Ag/SBA-15 (R), (c) Ag/SBA-15 (B), (d) $\mathrm{Pd} / \mathrm{Ag} / \mathrm{SBA}-15$ (Y), (e) $\mathrm{Pd} / \mathrm{Ag} / \mathrm{SBA}-15$ (R) and (f) Pd/Ag/SBA-15 (B) in dark conditions. Reaction conditions: catalyst (5 mg), $p$-NS $(5 \mathrm{~mL}, 20 \mathrm{mM}), \mathrm{AB}(5 \mathrm{~mL}, 0.06 \mathrm{M})$ and under an inert argon atmosphere at room temperature and atmospheric pressure.
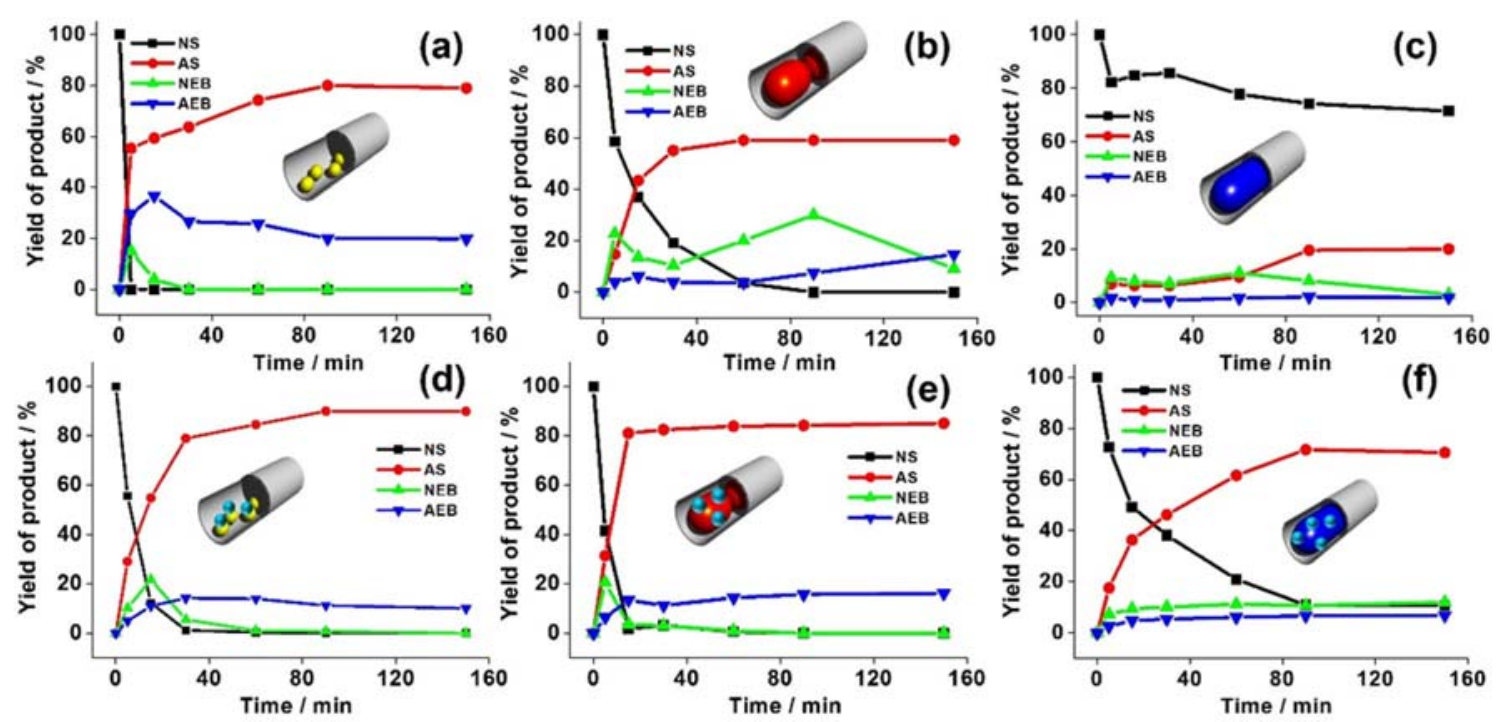

Figure 5. Chemoselective hydrogenation reaction of $p$-nitrostyrene ( $p$-NS) to $p$-aminostyrene ( $p$-AS) using (a) Ag/SBA-15 (Y), (b) Ag/SBA-15 (R), (c) Ag/SBA-15 (B), (d) Pd/Ag/SBA-15 (Y), (e) $\mathrm{Pd} / \mathrm{Ag} / \mathrm{SBA}-15$ (R) and (f) Pd/Ag/SBA-15 (B) under light irradiation conditions. Reaction conditions: catalyst $(5 \mathrm{mg}), p$-NS $(5 \mathrm{~mL}, 20 \mathrm{mM}), \mathrm{AB}(5 \mathrm{~mL}, 0.06 \mathrm{M})$ and under an inert argon atmosphere at room temperature and atmospheric pressure.

Under visible light irradiation, all catalysts showed enhancement in the catalytic activity especially in the production of $p$-AS owing to the Ag-LSPR effect, as shown in Figure 5. No products were observed even under light irradiation conditions in the absence of a catalyst. The conversion of $p$-NS over $\mathrm{Pd} / \mathrm{Ag} / \mathrm{SBA}-15$ (Y) reaches as high as $89 \%$ of $p$-AS at $90 \mathrm{~min}$ and
Ag/SBA-15 (B) showed an increment from 9.3\% (dark) to $19.5 \%$ conversion. The enhancement factor was calculated for all the catalysts by simple division of their conversion rate $\left(\% \mathrm{~min}^{-1}\right)$ in dark and under light irradiation conditions, which is summarized in Table S1 (Supplementary Information). The maximum enhancement (nearly twice) was shown by Ag/SBA-15 (B) and 


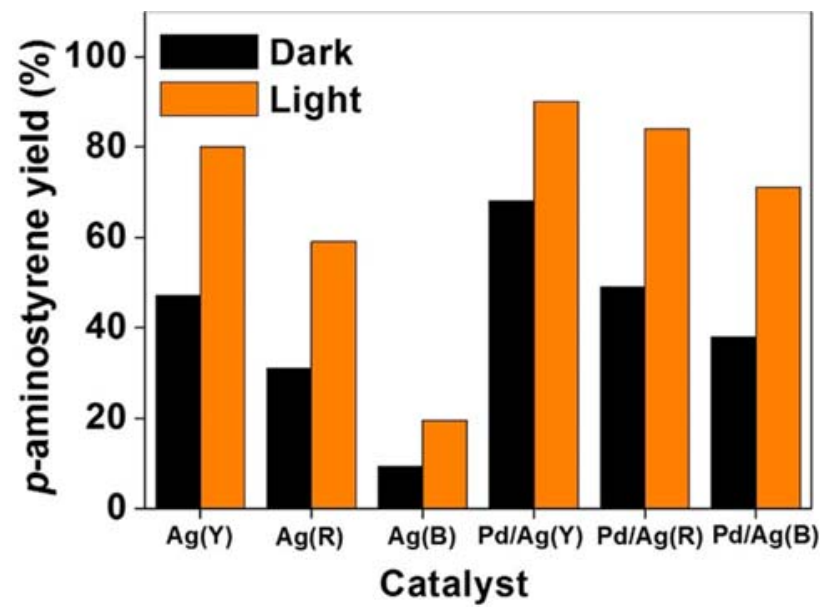

Figure 6. Comparison of yields of $p$-aminostyrene ( $p$-AS) for all catalysts in dark and under light irradiation conditions.

$\mathrm{Pd} / \mathrm{Ag} / \mathrm{SBA}-15$ (B) because of their maximum light absorption in the UV-vis spectra. The order follows the following trend: Ag/SBA-15 (B) (2.0) > Ag/SBA-15 (R) $(1.9)>\mathrm{Pd} / \mathrm{Ag} / \mathrm{SBA}-15$ (B) $(1.86)>\mathrm{Pd} / \mathrm{Ag} / \mathrm{SBA}-15$ (R) $(1.71)>$ Ag/SBA-15 (Y) (1.7) $>$ Pd/Ag/SBA15 (Y) (1.32). The values mentioned in parentheses are the enhancement values upon light irradiation conditions.

The higher selectivity under visible light irradiation can be due to the efficient transfer of $\mathrm{H}^{+} / \mathrm{H}^{-}$pair to the polar bonds in the $-\mathrm{NO}_{2}$ group of NS. The enhancement tendency was also found to be consistent with the $\mathrm{AB}$ dehydrogenation reaction. A comparison of $p$-AS conversion in dark and under light irradiation is summarized in Figure 6. It is easier to understand that the Ag/SBA-15 (Y) and $\mathrm{Pd} / \mathrm{Ag} / \mathrm{SBA}-15(\mathrm{Y})$ showed most active catalytic activity while Ag/SBA-15 (B) and Pd/Ag/SBA-15 (B) displayed maximum enhancement due to their broad absorption in the visible and near-IR region. It can be predicted that under visible light irradiation, charge polarization of plasmonic metal NPs leads to efficient adsorption of polar molecules onto them and catalyze the reduction by in situ synthesized gaseous $\mathrm{H}_{2}$. The synergistic effect of PdAg NPs enhances the efficient generation of $\mathrm{H}_{2}$ and plasmonic $\mathrm{Ag}$ leads to the efficient charge separation under visible light irradiation. The enhanced activity under visible light irradiation can be explained due to the generation of excited hot electrons. These electrons transfer to the adsorbate orbitals to form a transient negative ion (TNI), which reacts on the surface of a metal. The work function values of $\mathrm{Ag}$ $(4.3 \mathrm{eV})$ is lower than those of $\mathrm{Pd}(5.0 \mathrm{eV})$, which makes the positioning of its Fermi energy level lower than that of $\mathrm{Ag}$. Therefore, the electron transfer from $\mathrm{Ag}$ to $\mathrm{Pd}$ is feasible. ${ }^{39,40}$

\section{Conclusions}

With an objective to improve the selectivity of reaction and activity of plasmonic photocatalysts, in this paper, we concluded the synthesis, characterization, and catalytic reaction utilizing size and color controlled Ag and $\mathrm{Pd} / \mathrm{Ag}$ NPs supported on mesoporous silica. The NPs display improved chemoselective reduction of $p$-NS to $p$-AS under visible light irradiation. The maximum conversion efficiency of $89 \%$-AS can be obtained using Pd/Ag/SBA-15 (Y) NPs under visible light irradiation. The improvement in the selectivity of $p$-AS was observed almost twice in comparison to dark condition for Ag/SBA-15 (B) and Pd/Ag/SBA-15 (B). The library of catalysts needs to be explored more in order to achieve complete selectivity in the product formation by utilizing a broad spectrum of solar energy. The low quantum efficiencies and poor light absorption are still the challenges in order to overcome the low photocatalytic activity.

\section{Supplementary Information (SI)}

Sample images for all catalysts, HAADF-STEM image of Ag/SBA-15 (B), TEM micrographs for Pd/Ag/SBA-15 (Y), (R) (B), FT-EXAFS spectra and reaction rate conversions in dark and under light irradiation condition for catalytic reaction are available in supplementary information. Supplementary Information is available at www.ias.ac.in/chemsci.

\section{Acknowledgements}

The present work was partially supported by Grants-inAid for Scientific Research (Nos. 26220911, 26630409, 26620194, and T16K14478) from the Japan Society for the Promotion of Science (JSPS) and MEXT. We acknowledge Dr. Eiji Taguchi and Prof. H. Yasuda at the Research Center for Ultra-High Voltage Electron Microscopy, Osaka University, for their assistance with the TEM measurements. YK, KM and HY thank MEXT program "Elements Strategy Initiative to Form Core Research Center". PV would like to acknowledge Japan International Corporation Agency (JICA) for research fellowship (D-14-90271).

\section{References}

1. Brongersma M L, Halas N J and Nordlander P 2015 Plasmon-induced hot carrier science and technology Nat. Nanotechnol. 1025

2. Hou W and Cronin S B 2013 A review of surface plasmon resonance-enhanced photocatalysis Adv. Funct. Mater. 231612

3. Cheng $\mathrm{H}$, Kamegawa $\mathrm{T}$, Mori $\mathrm{K}$ and Yamashita $\mathrm{H}$ 2014 Surfactant-free nonaqueous synthesis of plasmonic molybdenum oxide nanosheets with enhanced catalytic activity for hydrogen generation from ammonia borane under visible light Angew Chemie Int. Ed. 532910 
4. Cheng H, Fuku K, Kuwahara Y, Mori K and Yamashita $\mathrm{H}$ 2015 Harnessing single-active plasmonic nanostructures for enhanced photocatalysis under visible light J. Mater. Chem. A 35244

5. Mitsudome T, Mikami Y, Matoba M, Mizugaki T, Jitsukawa K and Kaneda K 2012 Design of a silver-cerium dioxide core-shell nanocomposite catalyst for chemoselective reduction reactions Angew. Chem. Int. Ed. 51 136

6. Shil A K, Sharma D, Guha N R and Das P 2012 Solid supported $\operatorname{Pd}(0)$ : an efficient recyclable heterogeneous catalyst for chemoselective reduction of nitroarenes Tetrahedron Lett. $\mathbf{5 3} 4858$

7. Saha A and Ranu B 2008 Highly chemoselective reduction of aromatic nitro compounds by copper nanoparticles ammonium formate J. Org. Chem. 736867

8. Corma A, Serna P, Concepción P and Calvino J J. 2008 Transforming nonselective into chemoselective metal catalysts for the hydrogenation of substituted nitroaromatics J. Am. Chem. Soc. 1308748

9. Shimizu K I, Miyamoto Y, Kawasaki T, Tanji T, Tai Y and Satsuma A 2009 Chemoselective hydrogenation of nitroaromatics by supported gold catalysts: mechanistic reasons of size- and support-dependent activity and selectivity J. Phys. Chem. C 11317803

10. Furukawa S, Yoshida Y and Komatsu T 2014 Chemoselective hydrogenation of nitrostyrene to aminostyrene over Pd- and Rh-based intermetallic compounds ACS Catal. 41441

11. Corma A and Serna P 2006 Chemoselective Hydrogenation of Nitro Compounds with Supported Gold Catalysts Science 313332

12. Corma A, Concepción P and Serna P 2007 A different reaction pathway for the reduction of aromatic nitro compounds on gold catalysts Angew. Chem. Int. Ed. 467266

13. Beier M J, Andanson J M and Baiker A 2012 Tuning the chemoselective hydrogenation of nitrostyrenes catalyzed by ionic liquid-supported platinum nanoparticles $A C S$ Catal. 22587

14. Pisduangdaw S, Mekasuwandumrong O, Yoshida $\mathrm{H}$, Fujita S I, Arai M and Panpranot J 2015 Flame-made $\mathrm{Pt} / \mathrm{TiO} 2$ catalysts for the liquid-phase selective hydrogenation of 3-nitrostyrene Appl. Catal. A Gen. 490193

15. Imamura $\mathrm{K}$, Nakanishi $\mathrm{K}$, Hashimoto $\mathrm{K}$ and Kominami H 2014 Chemoselective reduction of nitrobenzenes having other reducible groups over titanium(IV) oxide photocatalyst under protection-, gas-, and metal-free conditions Tetrahedron 706134

16. Qian X, Kuwahara Y, Mori K and Yamashita H 2014 Silver Nanoparticles Supported on $\mathrm{CeO}_{2}-\mathrm{SBA}-15$ by Microwave Irradiation Possess Metal-Support Interactions and Enhanced Catalytic Activity Chem. A Eur. J. 2015746

17. Boymans E, Boland S, Witte P T, Muller C and Vogt D 2013 Chemoselective Hydrogenation of Functionalized Nitroarenes using Supported Mo Promoted Pt Nanoparticles ChemCatChem $\mathbf{5} 431$

18. Fujita S I, Watanabe H, Katagiri A, Yoshida H and Arai M 2014 Nitrogen and oxygen-doped metal-free carbon catalysts for chemoselective transfer hydrogenation of nitrobenzene, styrene, and 3-nitrostyrene with hydrazine J. Mol. Catal. A Chem. 393257
19. White R, Luque R, Budarin V, Clark J and Macquarrie D 2009 Supported metal nanoparticles on porous materials. Methods and applications Chem. Soc. Rev. 38481

20. Fuku K, Hayashi R, Takakura S, Kamegawa T, Mori K and Yamashita H 2013 The Synthesis of Size- and ColorControlled Silver Nanoparticles by Using Microwave Heating and their Enhanced Catalytic Activity by Localized Surface Plasmon Resonance Angew. Chem. Int. Ed. 527446

21. Linic S, Aslam U, Boerigter C and Morabito M 2015 Photochemical transformations on plasmonic metal nanoparticles Nat. Mater. 14567

22. Jain P K and El-Sayed M A 2010 Plasmonic coupling in noble metal nanostructures Chem. Phys. Lett. 487 153

23. Jain P K, Huang X, El-Sayed I H, El-Sayed M A 2008 Noble metals on the nanoscale: Optical and photothermal properties and some applications in imaging, sensing, biology, and medicine Acc. Chem. Res. 411578

24. Verma P, Kuwahara Y, Mori K and Yamashita H 2015 Synthesis and characterization of a $\mathrm{Pd} / \mathrm{Ag}$ bimetallic nanocatalyst on SBA-15 mesoporous silica as a plasmonic catalyst J. Mater. Chem. A 318889

25. Verma P, Kuwahara Y, Mori K and Yamashita H 2016 $\mathrm{Pd} / \mathrm{Ag}$ and $\mathrm{Pd} / \mathrm{Au}$ bimetallic nanocatalysts on mesoporous silica for plasmon-mediated enhanced catalytic activity under visible light irradiation J. Mater. Chem. A 410142

26. Mori K, Verma P, Hayashi R, Fuku K and Yamashita H 2015 Color-Controlled Ag Nanoparticles and Nanorods within Confined Mesopores: Microwave-Assisted Rapid Synthesis and Application in Plasmonic Catalysis under Visible-Light Irradiation Chem. Eur. J. 21 11885

27. Verma P, Kuwahara Y, Mori K and Yamashita H 2017 Enhancement of Ag-based Plasmonic Photocatalysis in the Hydrogen Production from Ammonia Borane by the Assist of Single-Site Ti-Oxide Moiety within the Silica Framework Chem. Eur. J. 233616

28. Shimizu K, Sawabe K and Satsuma A 2011 Unique catalytic features of $\mathrm{Ag}$ nanoclusters for selective NOx reduction and green chemical reactions Catal. Sci. Technol. 1331

29. Matsushima Y, Nishiyabu R, Takanashi N, Haruta M, Kimura H and Kubo Y 2012 Boronate self-assemblies with embedded Au nanoparticles: preparation, characterization and their catalytic activities for the reduction of nitroaromatic compounds J. Mater. Chem. 22 24124

30. Tuteja J, Nishimura S and Ebitani K 2014 Base-free chemoselective transfer hydrogenation of nitroarenes to anilines with formic acid as hydrogen source by a reusable heterogeneous $\mathrm{Pd} / \mathrm{ZrP}$ catalyst $R S C A d v .4$ 38241

31. Carrus M, Fantauzzi M, Riboni F, Makosch M, Rossi A, Elena S and Bokhoven J A 2016 Increased conversion and selectivity of 4-nitrostyrene hydrogenation to 4-aminostyrene on $\mathrm{Pt}$ nanoparticles supported on titanium-tungsten mixed oxides Appl. Catal. A Gen. 519 130

32. Xu Q and Chandra M 2006 Catalytic activities of nonnoble metals for hydrogen generation from aqueous 
ammonia-borane at room temperature J. Power Sources. 163364

33. Kominami H, Nakanishi K, Yamamoto S, Imamura K and Hashimoto K 2014 Photocatalytic deoxygenation of sulfoxides to sulfides over titanium(IV) oxide at room temperature without use of metal co-catalysts Catal. Commun. 54100.

34. Dhakshinamoorthy A, Alvaro M and Garcia H 2009 Metal-organic frameworks (MOFs) as heterogeneous catalysts for the chemoselective reduction of carboncarbon multiple bonds with hydrazine Adv. Synth. Catal. 3512271

35. Wei H, Liu X, Wang A, Zhang L, Qiao B, Yang X, Huang Y, Miao S, Liu J and Zhang T $2014 \mathrm{FeO}_{\mathrm{X}}-$ supported platinum single-atom and pseudo-single-atom catalysts for chemoselective hydrogenation of functionalized nitroarenes Nat. Commun. 55634

36. Sharma U, Verma P K, Kumar N, Kumar V, Bala M and Singh B 2011 Phosphane-free green protocol for selec- tive nitro reduction with an iron-based catalyst Chem. Eur. J. 175903

37. Blaser H U, Steiner H and Studer M 2009 Selective catalytic hydrogenation of functionalized nitroarenes: an update ChemCatChem 1210

38. Noronha R G, Romao C and Fernandes A C 2009 Highly chemo-and regioselective reduction of aromatic nitro compounds using the system silane/oxo-rhenium complexes J. Org. Chem. 746960

39. Verma P, Kuwahara Y, Mori K and Yamashita H 2017 Synthesis of mesoporous silica supported Ag nanorodsbased bimetallic catalysts and investigation of their plasmonic activity under visible light irradiation Catal. Sci. Technol. 72551

40. Verma P, Yuan K, Kuwahara Y, Mori K and Yamashita H 2017 Enhancement of plasmonic activity by Pt/Ag bimetallic nanocatalyst supported on mesoporous silica in the hydrogen production from hydrogen storage material Appl. Catal. B doi:10.1016/j.apcatb.2017.05.017. 